\title{
Acute viral bronchiolitis: Dawn of a new era for the prevention of respiratory syncytial virus infection through vaccination
}

Many cases of bronchiolitis are caused by the respiratory syncytial virus (RSV), which was first identified in 1956 as causing illness in humans. Despite ongoing efforts since the 1960s to develop an RSV vaccine, it has remained elusive. The RSV vaccine research agenda experienced a major setback after the increased susceptibility to severe RSV disease and death in children who received the first formalin-inactivated vaccine in the 1960s. Only in the mid-1980s was the search for an RSV vaccine re-ignited. Alternative approaches to developing this vaccine included attempts at attenuation of RSV, which generally resulted in vaccine candidates that were either too reactogenic or too attenuated. Furthermore, the targeted approach of using the conserved fusion protein (F-protein), although showing some promise in older persons with underlying medical conditions, was not developed into a potential candidate for young children, for whom the need is greatest.

The F-protein target, however, was used to develop palivizumab, a humanised monocloncal antibody that has been shown - since the mid-1990s - to prevent RSV lower respiratory tract infection in young children with underlying risk factors. However, the widespread use of palivizumab has been impeded by the logistics to provide monthly intramuscular injections for 4 - 5 months of the year while the RSV circulates in temperate climates, and by its cost, which makes it unaffordable and not cost-effective in most countries. Also, as it is only targeted at high-risk children, it misses prevention of RSV in otherwise healthy children, who comprise $70 \%$ of all RSV hospitalisations.

The prospect of more affordable and efficacious vaccines to prevent RSV illness in the majority of children has changed over the past 5 years. This includes the successful development of a re-engineered F-protein monoclonal antibody that has an extended half-life, which would allow for a single dose to provide protection against RSV illness for the duration of the RSV season (4 - 5 months). The first safety and antibody kinetics study of this monoclonal antibody was recently concluded. Moreover, a number of other RSV vaccine candidates $(>20)$ are currently being researched in clinical trials. ${ }^{[1]}$ These include the next generation of re-engineered live attenuated RSV vaccines, vector-based vaccines, F-protein-based subunit vaccines, including the use of nanoparticle technology or targeting the prefusion epitopes of the F-protein. ${ }^{[2,3]}$

The strategy to optimise the protection of young infants, considering that the mean age for contracting RSV is usually $4-5$ months and that these children might be too young to benefit from active vaccination, has also recently undergone a paradigm shift. In particular, it has been accepted that pregnant women may potentially be vaccinated to protect their infants against illness during the first 3 - 4 months of life by enhancing transplacental acquisition of vaccine-induced maternal antibody. This strategy not only contributed to the control of neonatal tetanus globally, but has more recently been shown to prevent influenza and pertussis in young infants in whom the burden of severe disease is the greatest. ${ }^{[4,5]}$ Accordingly, the first studies of the nanoparticle RSV F-protein vaccine candidate in pregnant women were recently completed, and a multicentre safety and efficacy trial is currently underway in pregnant women, also at multiple sites in South Africa. Furthermore, at least three other vaccines, also targeted at the F-protein, are likely to enter into clinical trials in pregnant women during the next 5 years.

Although vaccination of pregnant women is likely to benefit their young infants, this will probably need to be coupled with other vaccination strategies in children to provide protection until at least 12 - 24 months of age. Vaccination strategies specifically targeted at children are also being pursued over and above passive immunisation with a monoclonal antibody. This could include using the same vaccine candidates being evaluated in pregnant women and live attenuated vaccines, especially in older infants.

Therefore, while RSV currently remains the leading cause of hospitalisation for lower respiratory tract infection globally ( $\sim 30$ $40 \%$ of all cases), it is hoped that the next 10 years will see the face of paediatric practice change once again, if some of the current passive and active RSV vaccine candidates are licensed. The challenge for low- and middle-income countries would be to ensure that any such vaccines are affordable and timeously implemented into public immunisation programmes to reduce RSV-associated hospitalisation sequelae such as recurrent wheezing and death.

\section{S A Madhi}

Guest editor

Vaccinology, Faculty of Health Sciences, University of the Witwatersrand, Johannesburg; South African Medical Research Council: Respiratory and Meningeal Pathogens Research Unit, Johannesburg; Department of Science and Technology/National Research Foundation: Vaccine Preventable Diseases, Johnnesburg; and National Institute for Communicable Diseases, Johannesburg, South Africa

shabirm@nicd.ac.za

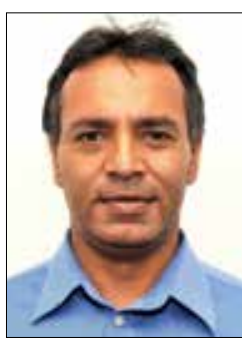

\section{R J Green}

\section{Guest editor}

Department of Paediatrics and Child Health, Faculty of Health Sciences, University of Pretoria, and Steve Biko Academic Hospital, Pretoria, South Africa robin.green@up.ac.za

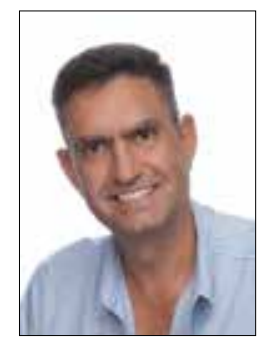

1. Mazur NI, Martinon-Torres F, Baraldi E, et al. Lower respiratory tract infection caused by respiratory syncytial virus: Current management and new therapeutics. Lancet Respir Med 2015;3(11):888-900. Jaberolansar N, Toth I, Young PR, Skwarczynski M. Recent advances in the development of subunitbased RSV vaccines. Expert Rev Vaccines 2016;15(1):53-68. DOI:10.1586/14760584.2016.1105134 Morrison TG, Walsh EE. Subunit and virus-like particle vaccine approaches for respiratory syncytial virus. Curr Top Microbiol Immunol 2013;372:285-306. DOI:1007/978-3-642-38919-1_14

4. Madhi SA, Cutland CL, Kuwanda L, et al. Influenza vaccination of pregnant women and protection of their infants. N Engl J Med 2014;371(10):918-931. DOI:10.1056/NEJMoal 401480

5. Amirthalingam G, Andrews N, Campbell $\mathrm{H}$, et al. Effectiveness of maternal pertussis vaccination in England: An observational study. Lancet 2014;384(9953):1521-1528. DOI:10.1016/S01406736(14)60686-3

S Afr Med J 2016;106(5):442. DOI:10.7196/SAMJ.2016.v106i5.10735 